\title{
Detection And Classification of Weft Knitted Fabrics Defects Using Gabor Wavelet
}

\author{
${ }^{1}$ Bassel A. El-Azab, ${ }^{2}$ Marwa Yasseen, ${ }^{3}$ Magdi Mohamed Fahmi, ${ }^{4}$ Heba Shalaby, \\ ${ }^{5}$ R.A.M. Abd El-Hady \\ ${ }^{1}$ Bachelor of Computer Science. Banha University. Egypt. \\ ${ }^{2}$ Ass. Lecturer, Spinning, Weaving \& Knitting Dep. \\ Faculty of Applied Arts, Banha University. Egypt. \\ ${ }^{3}$ Senior Consultant Engineer, Vice Chairman Riad Group. Egypt. \\ ${ }^{4}$ Professor of Textile Design, Spinning, Weaving \& Knitting Dep. \\ Faculty of Applied Arts, Banha University. Egypt. \\ ${ }^{5}$ Professor of Engineering \& Knitting Technology, Spinning, Weaving \& Knitting Dep. \\ Faculty of Applied Arts, Helwan University. Egypt.
}

\begin{abstract}
The globalization of competition, the complexity of the economy and the plethora of information available today place companies in a more than shifting context with which they must cope. Accelerating change becomes a constant feature of business life. Companies need methodological help to process a lot of information, In today's competitive world, customers are demanding better quality products with fast and reliable deliveries. To meet this demand, new manufacturing technologies are developing rapidly, resulting in new products and improvements in manufacturing processes.

Today's challenging world demands minimum loss and waste from industries. Moreover, it has to ensure the required quantity and quality with customer delivery lead time. A Circular weft knitting machine contains different parts such as needles, cams, sinkers, Fabric takedown mechanism, creel, a yarn metering and storage device, yarn breakage indicator, feeders and lubrication system. All those machine parts are responsible to increase or decrease the productivity of weft knit fabric production as well as the fabric quality. The Circular weft Knitting Machine has to stop when defects occurred and then faults are corrected, which results in a loss in time and efficiency in order to be ready to meet customer requirements; the goal is to quickly provide products that combine quality and competitive price. In this sense, effective monitoring is required to avoid defects and maintain high productivity and customer required quality. The purpose of this study is to identify and analyze weft knitted fabric defects on the weft circular knitting machine of knitting industries.

This paper describes a computer vision-based fabric inspection system implemented on a weft circular knitting machine to detect defection and classification of the weft-knitted fabric defects under construction. We using Gabor Wavelets that have been successfully applied to various machine vision applications such as Texture segmentation, Edge detection, and Boundary detection, a multi-scale and multi orientation Gabor filter scheme simulates the human eye and that's applied to the weft-knitted fabric under construction. On-line weft knitted fabric defect detection was tested automatically by analyzing fabric images captured by a digital camera using Gabor wavelets and classification (Identification) these fabric defects to known classes. We succeeded to detect weft knitted fabric defects and classify defect's on the weft circular knitting machine at the same time the machine stops to correct the fabric defect to achieve customer required quantity and quality. As well as we cancel the fabric inspection process that means, saving money, time, manpower which leads to reducing production lead time and cost.
\end{abstract}

Keywords: Gabor Filters, Circular weft knitting machine, Computer vision, Classification, Fabric faults, Feature Extraction, Plain Structure.

\section{INTRODUCTION}

Knitting is the second most frequently used method of fabric construction. The term "Knitting" describes the technique of constructing textile structures by forming a continuous length of yarn into columns of vertically intermeshed loops. Knitted fabrics have been gaining popularity during the past two decades, thanks to the increased versatility of techniques and adaptability of the 
many new manmade fibers. Knitted fabrics are now widely used in the applications where woven fabrics formerly predominated. Today, the usage of knitted fabrics ranges from hosiery, underwear, sweaters, slacks, to rugs and other home furnishings [1].

Most single-jersey (plain) fabric is produced on weft knitting circular machines whose latch needle cylinder and sinker ring revolve through the stationary knitting cam systems that, together with their yarn feeders, are situated at regular intervals around the circumference of the cylinder. The yarns is supplied from cones, placed either on an integral overhead bobbin stand or on a free-standing creel, through tensioners, stop motions and guide eyes down to the yarn feeder [2]. Plain weft knit, the basic form of knitting can be produced in flat knit or in tubular (or circular) form. It is also called jersey stitch or balbriggan stitch. A row of latch or beard needles is arranged in a linear position on a needle plate or in a circular position on a cylinder. These fabrics have the tendency of curling up at the edges which is controlled to a level through certain finishes. Plain-knit fabrics stretch more in the width than in the length and as such, they are widely used for making underwear, gloves, hosiery and sweaters [3].

Quality is essential for any manufacturing or service industry to guarantee sufficient market share and meeting customer satisfaction there by to win customer loyalty. In fact, in today's very competitive market, it can be said that quality is the main factor in determining the success or failure of an organization. [4]. A defect in the weft knitted fabrics is an abnormality, which spoils the aesthetics i.e. the clean \& uniform appearance of the fabric \& effects the performance parameters, like; dimensional stability etc. There are various types of defects, which occur in the weft knitted fabrics of all types, caused by a variety of reasons. The same type of defects may occur in the fabric, due to a variety of different causes e.g. Drop Stitches, Spirality. Prime causes of the fabric defects are, as follows [5, 6];

- Yarns.

- Knitting Elements.

- Knitting Machine Settings.

- Dyeing.

- Finishing.

As regard to greige weft knit fabric, the first three causes are the sources for defects to occur. Weft knitting fabric defects are those that can be created during the fabrication (fabric formation) and some of them are listed below. These defects are checked during the quality control process [5] ;

- Needle line: when the needle is broken, bent, and old or damaged on the knitting machine it creates the straight line mark on the length of the fabric.

- Sinker line: when the sinker is broken, bent, and old or damaged on the knitting machine it will create strait line mark on the length wise direction of the fabric.

- Stripe mark/Bar defect: this defect is created when thin and thick yarns are mixed, when the tension of one yarn is varied from the others, due to count variation, when the origins of the cotton fiber from which yarns are made are different.

- Canal mark: is the straight line mark through the length of the fabric and can be easily visible on the fabric. Can be occurred due to the needle adjustment problem.

- Different tears and holes on the fabric: this can be occurred due to the needle breaks, yarn breaks or other.

- Grease and oil stain: this defect arises due to the improper fabric handling of the griege fabric or if the machine is not properly cleaned. It might or might not be removed after washing.

- Nep, thick, thin place on the fabric: this defect is due to the irregularity of yarn used for knitting. This irregularity of yarn will cause the dyeing problem usually dye absorption problem (dark and light shade) making the stripe on the fabric.[5,6]

As knitting is the process of converting yarns to fabric, during the process defects like needle-line, hole, yarn variation, lycra jump, oil stain, sinker mark and so on are appeared and because of these defects the rejection rate reaches to $7.87 \%$ and cost of money loss and it also damages the image of any company. This rejection rate is caused due to various reasons such as yarn tension, machine dust, worn-out knitting element (sinker, needle, feeder), air condition, yarn imperfection, misalignment of yarn feeder, damaged cylinder cut, blockage of oil hose, machine jerky. Therefore the required quality standards (parameters) will not be fulfilled as per the customers 'demand and to avoid this rejection rate of fabric, it is necessary to avoid defects by prioritizing them [7].

Industrial inspection problems are highly complex because they require real time solution. One of the most challenging industrial inspection problems is defect detection in weft knitted fabrics and classifies it. Any variation to the knitting process needs to be investigated and corrected. A great many methods of texture analysis have been investigated during the past several decades. Textured materials, such as woven and knitted fabrics, possess strong periodicity due to the repetition of the basic weaving pattern [8]. Textured materials take many forms and while there is a remarkable similarity in overall automation 
requirements for visual inspection, the cost-effective solutions are application specific and generally require extensive research and development efforts [9]. The detection of local fabric defects is one of the most intriguing problems in computer vision and has received much attention over the years [10]. A series of algorithms on the basis of software are presented in fabric detection now. There are into, three general types of fabric detection approaches are, respectively, based on the statistical, the model and the spectral methods [11]. Gabor filter is especially effective for fabric defect detection. Gabor filter is essentially a band-pass filter. In the spatial domain, its impulse response, Gabor function, is the product of a complex exponential function and a Gaussian function. Gabor filter has high resolution capability both in spatial domain and in frequency domain [12].

The family of 2-D-Gabor filters was originally presented by Daugman [13] as a framework for understanding the orientation selective and spatial frequency selective receptive field properties of neurons in brain visual cortex. Defect detection in produced weft knitted fabrics (plain structure) is done using Fourier transform, Wavelet transform, Gabor filters and other methods. When using Gabor filter bank for defect detection, the problems we meet are that the more filters in filter bank, the longer it needs to realize defect segmentation, and some filter in filter bank may be bad for the final defect detection result [12]. We use gabor filter bank and proposed method to select proper filter for segmented faults. We are using feature extraction from response image to classification (Identification) segmented fault.

This research aims to address the above quality problem of knitting section by identifying the $80 \%$ causes of quality defects and proposing the possible solution for the critical ones. After the data is analyzed, the three critical fabric defects (Broken Yarn or Needle line, Hole, Yarn variation) are achieved and these fabric defects need to be corrected to minimize the rejection rate by $80 \%$. We succeeded to detect these defects and classify defect's and stop the circular weft knitting machine to achieve better quantity and quality.

\section{MATERIALS AND METHDS}

\subsection{Single Jersey Machine Specification}

Single jersey knitting machine was selected. The target sample was single jersey plain structure. Table (1) shows the Single jersey machine specification that used to produce fabric samples under study.

Table (1) The Single jersey Machine Specification

\begin{tabular}{|l|l|}
\hline Company & Mayer \& cie \\
\hline Machine's type & Single jersey \\
\hline Machine's Model & GL 615 S \\
\hline Made in & Germany \\
\hline Year of made & 2006 \\
\hline Construction & Single jersey \\
\hline Machine gauge (Needle / Inch) & 28 \\
\hline No. of Needles & 2988 \\
\hline Cylinder diameter (Inch) & $34 "$ \\
\hline Yarn Count & $30 / 1$ Ne Indian combed cotton \\
\hline Stitch Length & 2.75 mm \\
\hline Fabric weight & 135 gsm \\
\hline Number of feeders & 108 \\
\hline Max speed & 25 \\
\hline
\end{tabular}

\subsection{Tools And Techniques}

For experimental work we need to use the following tools and techniques;

- Laptop (processor: Intel core I3 with 2.53GH4, AMD.

- Graphic DRM 2.50) 4G memory.

- Arduino kit (for controlling the circular weft knitting machine).

- Python programming language.

- $\quad$ Scikit- learn (machine learning package).

- Opencv (computer vision python package).

- Proposed algorithm (locate defects). 
International Journal of Advances in Scientific Research and Engineering (ijasre), Vol 6 (2), February-2020

\subsection{Gabor Filter}

A 2-D Gabor function $\mathrm{g}(\mathrm{x}, \mathrm{y})$ is a 2-D gaussian modulated (Fig.1 (a)) with a complex exponential (sinusoidal) (Fig.1 (b)), and is given as,

$$
g(x, y)=\frac{1}{2 \pi \sigma_{x} \sigma_{y}} \exp \left(\left[-\frac{1}{2}\left(\frac{x^{\prime 2}}{\sigma_{x}^{2}}+\frac{y^{\prime 2}}{\sigma_{y}^{2}}\right)\right]\right) \cdot \exp \left(j 2 \pi W x^{\prime}\right)
$$

$\sigma_{\mathrm{x}}$ and $\sigma_{\mathrm{y}}$ define the Gaussian envelope along the $\mathrm{x}$ and y axes. If $\sigma_{\mathrm{x}}=\sigma_{\mathrm{y}}$, the Gabor filter is circularly symmetric [14]. And, $\mathrm{W}$ is called the modulation frequency and

$$
\begin{aligned}
& x^{\mathrm{j}}=x \cos (\theta)+y \sin (\theta) . \\
& y^{\mathrm{j}}=y \cos (\theta)-x \sin (\theta) .
\end{aligned}
$$

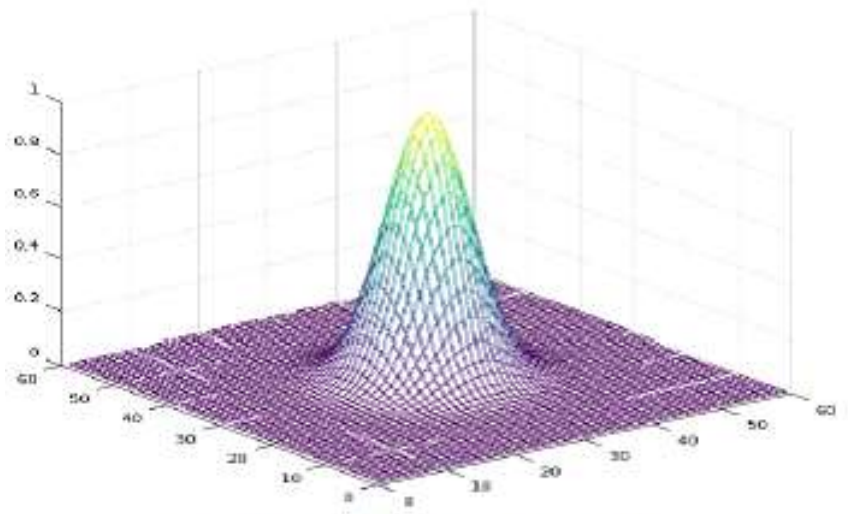

(a) $3 \mathrm{D}$ representation of a Gaussian mask

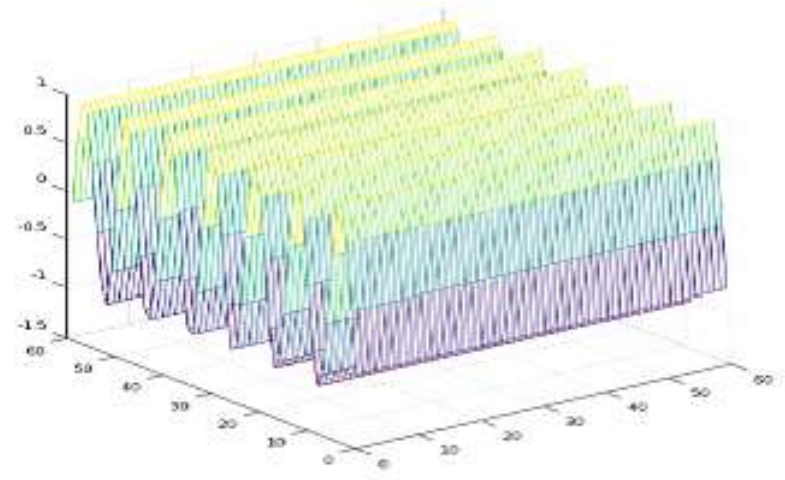

(b) $3 \mathrm{D}$ representation of sinusoid with $\mathrm{W}=0.5$ and Sigma $=5$ and theta $=0$

Fig. (1) Gabor Filter

For a given image $\mathrm{T}(\mathrm{x}, \mathrm{y})$, the discrete Gabor wavelet transform can be represented as given in Eq. (2).

$$
\left.G_{p q}(x, y)=\left[g_{p q}^{e} * T(x, y)\right]^{2}+\left[g_{p q}^{o} * T(x, y)\right]^{2}\right]^{\frac{1}{2}}
$$

where, $\mathrm{p}$ and $\mathrm{q}$ represents the scale and the orientation.

The symbol * denotes a 2-D convolution, and $\mathrm{g}_{\mathrm{pq}}^{\mathrm{e}}$ and represent the real (even) and the imaginary (odd) parts respectively, of the Gabor filter $\mathrm{g}_{\mathrm{pq}}^{\mathrm{o}}(x, y)$. General responses of the real part and the imaginary part from 2-D Gabor function are exhibited in Fig.2. 

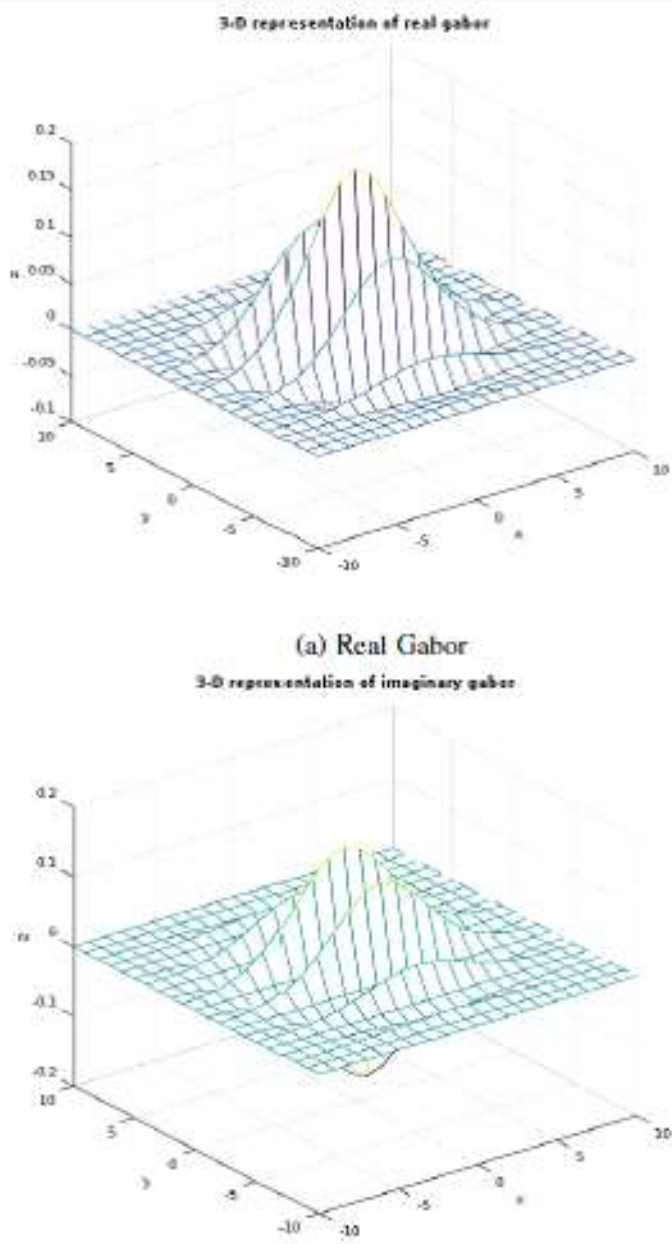

(b) Imaginary Gabor

Fig. (2) Outline Drawing for Basic Gabor Function

\subsubsection{Improved Gabor Filter}

The computation required for Gabor Wavelet based feature extraction is very intensive. This in turn creates a bottleneck problem for real time processing. Hence, an efficient method for extracting Gabor features is needed for many practical applications [15].

Wei Jiang et al. [16] have proposed that the imaginary part of a Gabor filter is an efficient and robust means for edge detection. The imaginary part of a Gabor Wavelet is as in Eq. (3).

$S(x, y)=\exp \left(\left[-\frac{x^{2}+y^{2}}{2 \sigma^{2}}\right]\right) \cdot \sin ([W \cdot(x \cos (\theta)+y \sin (\theta))])$

Edges can be detected by using this simplified Gabor Wavelet. Set of Simplified Gabor kernels can be obtained from Eq. (3) by selecting different center frequencies and orientations. These kernels are used to extract features from an image. This method is known as Simplified Gabor wavelet [15].

\subsubsection{Local Defect Using Gabor Filter}

Segmentation of a similar class of local weft knitted fabric defects with a priori knowledge about the orientation and size of a sample defect can be regarded as supervised defect segmentation [10]. When the approximate orientation and size of defects are known, the power spectrum sampling of the respatial frequency plane is not necessary. Fabric defect is of strong orientation and different scale so it can react remarkably to certain appropriate Gabor filters. In such cases, the segmented has been achieved with only one Gabor filter, from the Gabor filter bank that can provide best discrimination of texture features against the defects [10]. Here, we use an easy and effective method proposed by Kumar [10] to choose an optimal filter to best describe the defect.

\section{A. Selection Proper Gabor Filter}

The defect detection algorithm is divided into many steps:- 
- A gabor filter bank of $\mathrm{p}^{*} \mathrm{q}$ that described above with $\mathrm{p}$ scales and $\mathrm{q}$ orientations is investigated for supervised defect detection.

- $\quad \mathrm{T}(\mathrm{x}, \mathrm{y})$ test sample image under inspection is divided into $\mathrm{K}$ non-overlapping square regions of size ( $\mathrm{R} * \mathrm{R}) \mathrm{pixels}$. Each of the filter bank from $\mathrm{i}=1$ to $\mathrm{p}^{*} \mathrm{q}$ in gabor filter bank is applied to number of divided regions image from $\mathrm{i}=1$ to $\mathrm{K}$ nonoverlapping square.

- Then we compute the average output of each square regions form $\mathrm{i}=1$ to $\mathrm{K}$ non-overlapping as in Eq. (4).

$$
D_{R}^{i}=\frac{1}{R+R} \sum_{(x, y) \in R} S_{p q}(x, y)
$$

Then we compute a cost function for each filter $\mathrm{i}=1$ to $\mathrm{p}^{*} \mathrm{q}$ in gabor filter bank with the maximum average output $\mathrm{D}^{\mathrm{i}}$ max and the minimum average output $\mathrm{D}^{\mathrm{i}}{ }_{\min }$ among all of the square regions.

$$
J(i)=\left(\frac{D_{\max }^{i}-D_{\min }^{i}}{D_{\min }^{i}}\right)
$$

In the last step we have number of $\mathrm{p}^{*} \mathrm{q}$ of cost function $\mathrm{J}(\mathrm{i})$ in Eq. (5) and we choose from $\mathrm{J}(\mathrm{i})$ the maximum value (highest $3 \operatorname{cost}$ function $\mathrm{J}_{\mathrm{rp}}$ ) which is the best representative filter to detect a class of local fabric defects under consideration.

$$
J_{r p}=\max _{1 \leq i \leq p * q}[J(i)]
$$

Here we now know the proper filter is applied to test sample image $\mathrm{T}(\mathrm{x}, \mathrm{y})$ to local defect detection and denoted as Tout (x; $\mathrm{y}$ ) then we segmented the defect using thresholding to binarization $\operatorname{TOut}(\mathrm{x} ; \mathrm{y})$.

\section{B. Segmentation}

The optimal result which is a gray image is to be converted into a binary image [8]. The thresholding limit is determined from a reference or defect-free fabric image $\mathrm{R}(\mathrm{x}, \mathrm{y})$ [10] which is clear image, image with no defects.

The process of image segmentation is based on image that hasn't defect (free image) on selecting proper gabor filter that described above we apply it to reference (defect-free fabric) image $\mathrm{R}(\mathrm{x}, \mathrm{y})$ to choose the maximum value of gray level within a window "W" that used it in binary image as in Eq. $(7,8)$.

$$
\begin{aligned}
& \text { ThresholdV alue }(\max ) \mathcal{T}_{\max }=\max _{x, y \in W}|R(x, y)| \\
& \text { ThresholdValue(min) } \mathcal{T}_{\min }=\min _{x, y \in W}|R(x, y)|
\end{aligned}
$$

"W" is a window centered at the image. The window size "W" is chosen to avoid any possible distortion effects from the image due to discontinuities at the border [6]. 1) binarization: The binary process of Binary image B(x,y) can be conducted as the following step:

$$
B(x, y)= \begin{cases}1, & \text { if } T_{\text {Out }}>\mathcal{T}_{\max } \text { or } T_{\text {Out }}<\mathcal{T}_{\min } \\ 0, & \text { otherwise }\end{cases}
$$

We use median filter after binarization process to remove noise from images. 


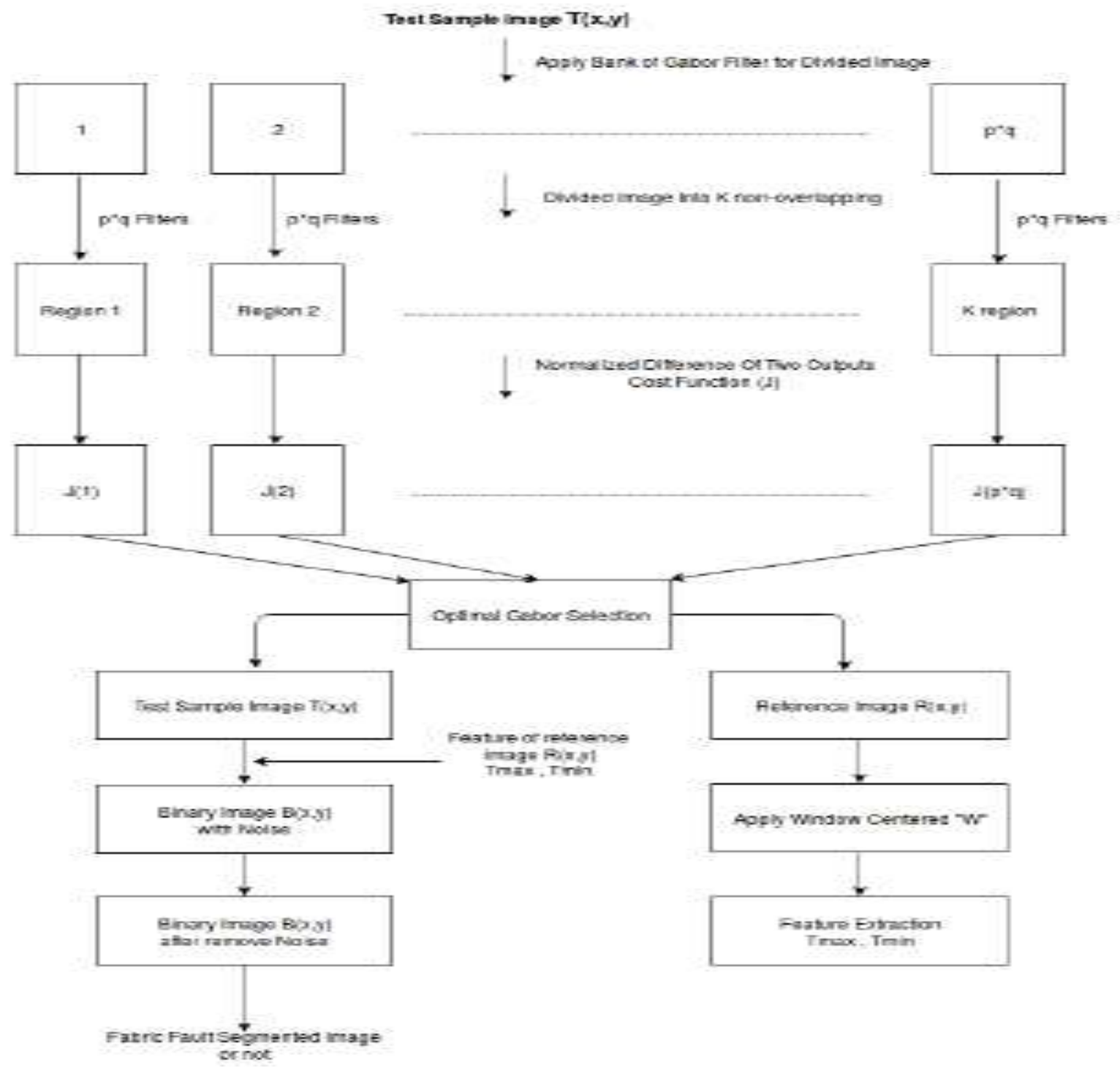

Fig. (3) Schema of Local Defect Detection

\section{RESULTS AND DISCUSSION}

Each Filter in the filter bank is implemented as a $5 * 5$ convolution mask for imaginary components. the gabor filters bank distributed at four scales and four orientation, number of orientations.

Where $W=0.5 * 2^{i-4}, \theta=(j-1) * \frac{\pi}{4}$,

For each $i=1,2, \ldots ., p$ number of scales and $j=1,2, \ldots ., q$

We accept images of fabric in circular knitting machine with resolution $(320 * 240)$ pixels, then we digitized images into resolution of $(256 * 256)$ pixels, with 8-bit resolution (256 gray levels).

Each of these images was divided into $\mathrm{K}$ non-overlapping where, $\mathrm{K}=16$ regions of 64_64pixels. Each of filter in bank gabor filters is applied into these regions and optimal gabor filter selection as described in section IV-A.

Fig.4 shows the final results of some Samples applied algorithm to local defect. There are four samples shown in Fig. 4 after applying proposed method for local defect detection. Fig. 5 shows the relation between number filter in bank gabor filters and corresponding cost function. 
International Journal of Advances in Scientific Research and Engineering (ijasre), Vol 6 (2), February-2020

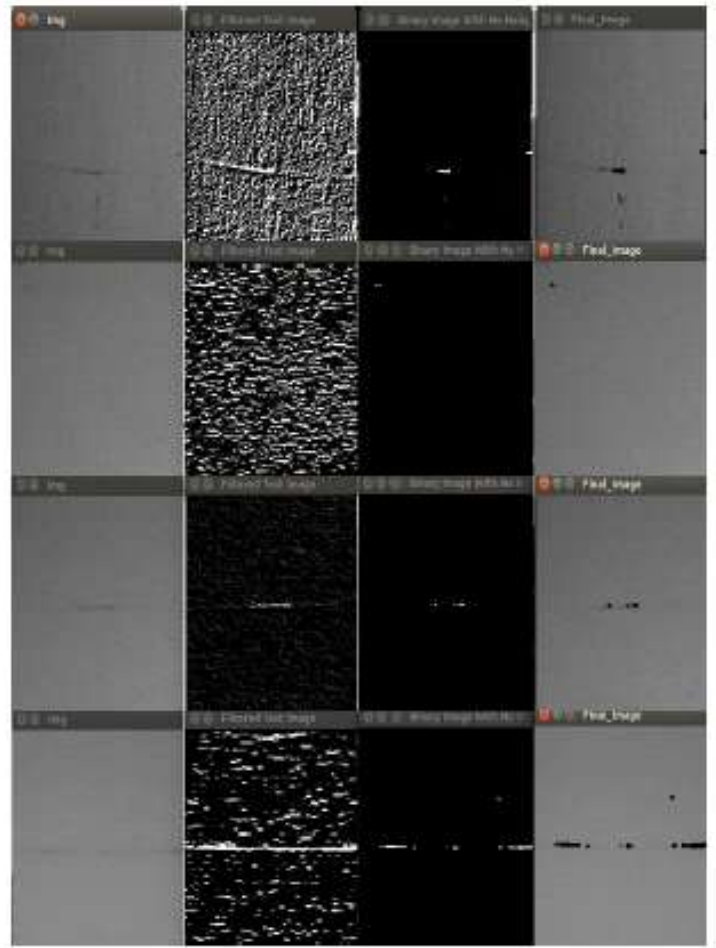

Fig. (4 ) Fabric Sample with Defect of Four Types [FD1; FD2; FD3; FD4]

First Column Respectively; Corresponding Optimal Result using Gabor Filters Second Column; Binary Image

Third column; Locate Defect in the Final Image

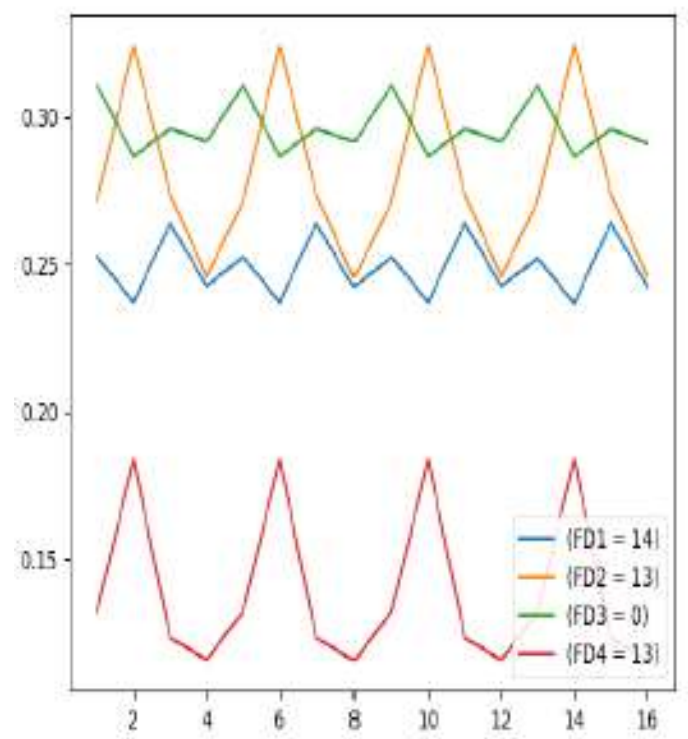

Fig. (5) Relation Between Filter Number i in $x{ }^{--}$axis and Cost Function $J(i)$ in $y^{-"}$ axis

As seen in Fig.5 the number of gabor filter equal to 16 on the horizontal axis, where number of scale $=\mathrm{i}=1 ; 2 \ldots ., \mathrm{p}$ and number of orientation $=j=1,2, \ldots ., q$.

Samples [FD1; FD2; FD3; FD4] achieve their peaks in the number 14, 13, 0 and 13 filter respectively.

Taken FD2 as an example, the index of optimal gabor filter that achieved highest cost function is 13 which means this optimal filter is in the 4rd scale and 1 st orientation.

Taken FD3 as an example, the index of optimal gabor filter that achieved highest cost function is 0 which means this optimal filter is in the 1rd scale and 1st orientation. In this part, several factors may influence the performance of segmentation. The frequency range of the Gabor filter bank is required to cover defects as much as possible [8]. 


\subsection{Defect Classification Using Logistic Regression}

In the last stage we select optimal gabor filter to locate defects in test images and after knowing the optimal gabor filter we applied this filter To the reference image $R(x, y)$ and the test image $T(x, y)$ and extract features from $R(x, y)$ to convert test image $\mathrm{T}(\mathrm{x}, \mathrm{y})$ to binary image to locate fabric defect based on threshold value extracted from $\mathrm{R}(\mathrm{x}, \mathrm{y})$.

Now we want to classify These types of defects And which class belongs to (Broken Latch/ Needle), (Hole), (Yarn variation /Wabra), etc and other types of fabric defects. After completing the last stage we could extract features from test images and use Logistic Regression as a classifier.

\subsubsection{Feature Extraction}

As in [8] they used 5 features called mean, variance, 1-norm, 2-norm and entropy to characterize the information of texture pattern.

$$
\begin{gathered}
F_{\text {mean }}^{i}=\frac{1}{p \cdot q} \sum I_{i}(x, y) \\
F_{\text {var }}^{i}=\frac{1}{p * q} \sum\left(I_{i}(x, y)-F_{\text {miean }}^{i}\right)^{2} \\
F_{\text {norm } 1}^{i}=\frac{1}{p \cdot q} \sum\left|I_{i}(x, y)\right| \\
F_{\text {norm } 2}^{i}=\frac{1}{p * q} \sum\left|I_{i}(x, y)\right|^{2} \\
F_{\text {entropy }}^{i}=\frac{1}{p * q} \sum\left|I_{i}(x, y)\right|^{2} \log \left|I_{i}(x, y)\right|^{2}
\end{gathered}
$$

We extract features from sample defect images after applying optimal gabor filter to use it in the trained data with classifier to predict type of fabric defect later.

\subsubsection{Logistic Regression Based Classification}

In the first, logistic regression model is binary classification. However, we can also use flavors of logistic to tackle multiclass classification problems, e.g., using the One-vs-All or One-vs-One approaches, via the related softmax regression / multinomial logistic regression [17].

The logistic function_(t) is defined as follows:

$$
\sigma_{(t)}=\frac{e^{t}}{e^{t}+1}=\frac{1}{1+e^{-t}}
$$

And the logistic function can now be written as:

$$
p(x)=\frac{1}{1+e^{-\left(\beta_{0}+\beta_{1} x\right)}}
$$

For more information see Ref [18].

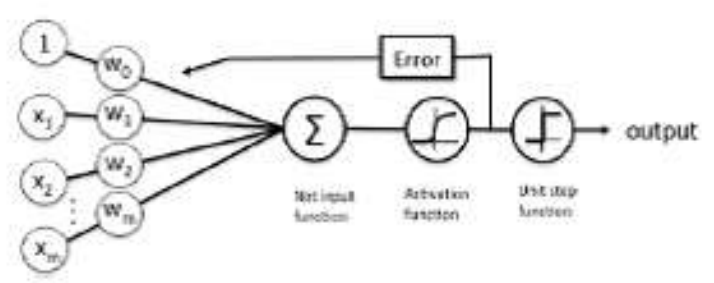

Fig. (6) Schematic of a Logistic Regression Classifier

\subsection{Results of Defect Detection Classification}

There are many types of fabric defects that appears when circular knitting machine working. We worked on three types of defects to classify it which are, (Broken yarn/needle), (Hole), (Yarn variation / Wabra). 
We have collected the data of three types to train it in classifier where,

- $\quad$ Type of defect (Hole). Number of defects $=480$.

- $\quad$ Type of defect (Broken yarn/latch). Number of defects $=355$.

- $\quad$ Type of defect (Yarn variation /Wabra). Number of defects $=875$.

After applying proposed method and image segmentation in order to locate fabric defects or found that no defects then extracting features from collected images And trained data with logistic regression classifier as mentioned above. We test new sample images to predict defects type and know type or defects class or classify it to unknown class but locate defects in both cases.

In the next figures, Fig. 7, Fig. 8, Fig. 9, showing sample images taken by webcam when circular weft knitting machine is working and locate defects, classify defects and send signal to arduino to stop machine.

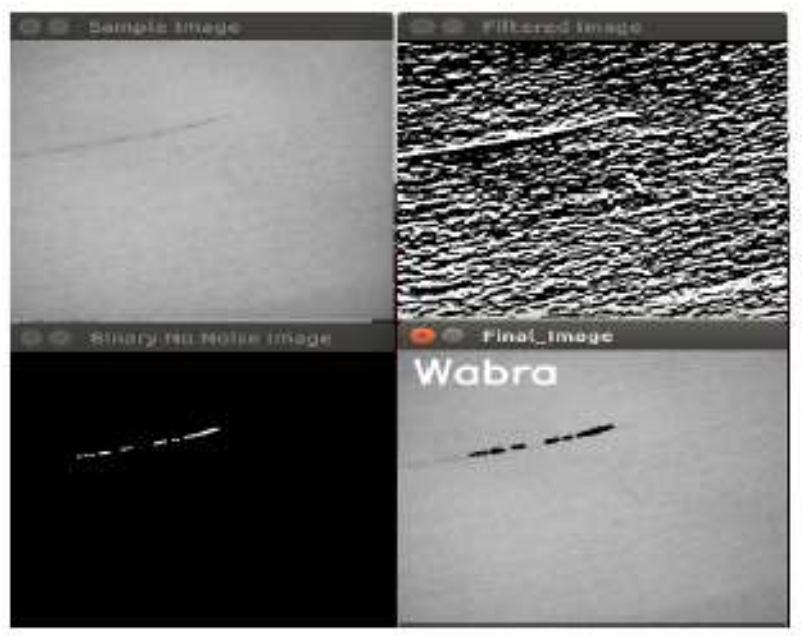

Fig. (7) Result of Type Defect (Yarn Variation /Wabra)

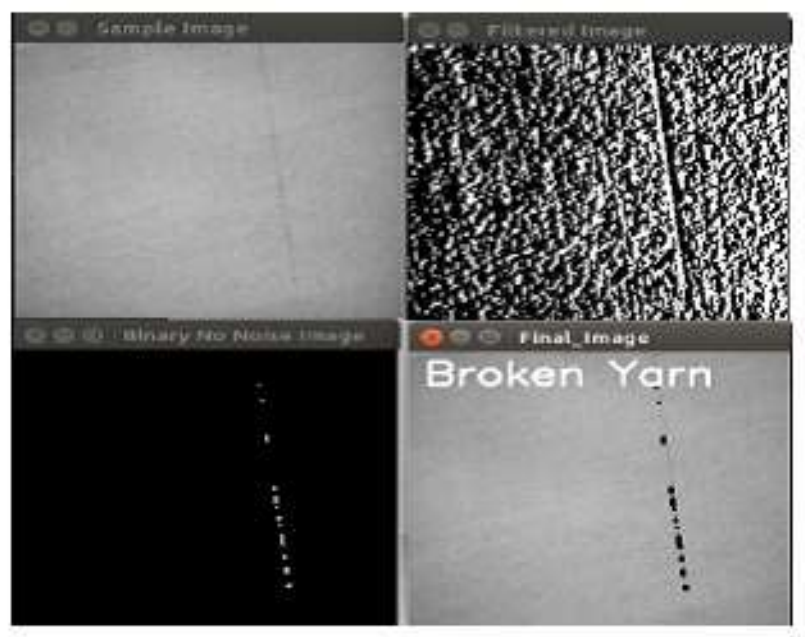

Fig. (8) Result of Type Defect (Broken Yarn/Needle) 


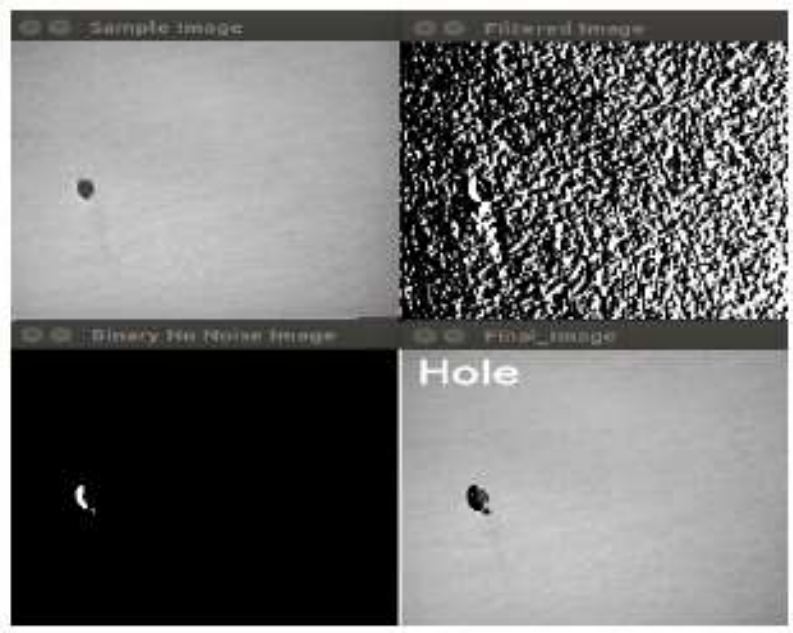

Fig. (9) Result Of Type Defect (Hole)

Fig. 7, Fig. 8 \& Fig. 9 showing four image for each type of fabric defects, First images are called (Sample Image) image taken by webcam when circular knitting machine is working and applying proposed algorithm described in this paper to defect detection and classification to stop machine if there is defect in fabric. Second images are called (Filtered Image) the result image of applying optimal gabor filter described in section IV-A. Third image called (Binary No Noise Image) the binarization process after applied optimal gabor filter described in section IV-B and After applying median filter to remove noise from binary image. Fourth image called (Final Image) the Final Result of image if there is defect locate and classify it as in Fig. 7, Fig. \& 8 Fig. 9 and if the image is clear with no defect the result become as in Fig. 10

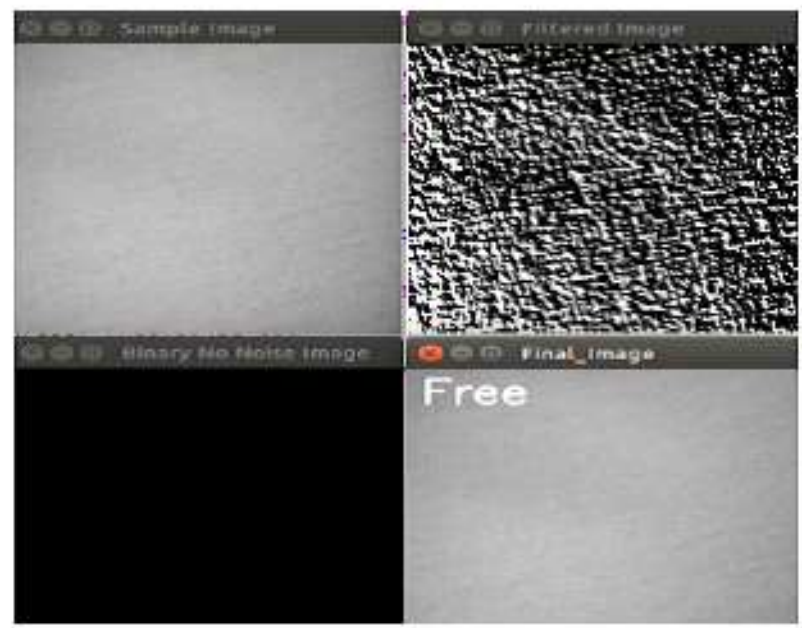

Fig. 10: Result of No Defect

\subsection{Approach}

We fixed the one-end of the webcam in the pipe (in the middle of the circular weft knitting machine) on specific area of the fabric and the second-end is connected to the laptop to allow it capture images then letting the circular knitting machine to work and then applying our algorithm (proposed algorithm) in order to defect detection and classification. If the proposed algorithm found a defect in the fabrics our python program will send a signal to out kit (Arduino kit) then the Arduino send signal to relay (that control the machine) and stop the machine and in case the fabric is clean with no defects the python program detect that and print message that said the fabric with no defects (the ideal case) and this process only success with three types of defects as mentioned above.

\subsubsection{Obstacles}

- On applying proposed method the result is not accurate $100 \%$ in defect detection and classification and this is because of the low quality of the webcam and also because of the machine vibrations the images are not perfectly clear and also because of the external factors such as illuminating the classification score is just $70 \%$.

- In classification also the results in not very accurate as features in final are very close to each others depends on fabric defects such as the small hole of fabric is closed to the defect caused by Yarn variation /wabara and the Yarn variation /wabara also sometimes close to value of broken yarn and so on. 


\section{CONCLUSIONS}

Quality is essential for any manufacturing or service industry to guarantee sufficient market share and meeting customer satisfaction there by to win customer loyalty. In fact, in today's very competitive market, it can be said that quality is the main factor in determining the success or failure of an organization. A defect in the weft knitted fabrics is an abnormality, which spoils the aesthetics i.e. the clean \& uniform appearance of the fabric \& effects the performance parameters, like; dimensional stability etc. There are various types of defects, which occur in the weft knitted fabrics of all types, caused by a variety of reasons.

This research aims to address the above quality problem of knitting section by identifying the $80 \%$ causes of quality defects and proposing the possible solution for the critical ones. After the data is analyzed, the three critical fabric defects (Broken Yarn or Needle line, Hole, Yarn variation) are achieved and these fabric defects need to be corrected to minimize the rejection rate by $80 \%$.

In general this project is aims to locate, classify defect of fabrics in order to control the circular weft knitting machine make it take action on different cases the first case if it has a fabric that have defects its action must to be stop as the fabric is not valid it has defects and this is done by our python code and the Arduino kit that connected to the relay to control our machine to make it smart and the second case that it has a clean fabric that accepted to be a texture and so the machine will not forced to stop it will continue its work We can improve the selection of optimal gabor filter by Genetic Algorithm as in [12]. We succeeded to detect these defects and classify defect's using log-gabor [19] and neural network as classifier and stop the circular weft knitting machine to achieve customer required quantity and quality. As well as we cancel fabric inspection process that means saving money, time, manpower which lead to reducing production lead time and cost?

\section{REFERENCES}

[1] D.J. Spencer.” Knitting technology (third edition)”, Wood head publishing limited, UK. 2001.

[2] Iyer, Mammel \& Schäch., "Circular knitting : technology, process, structures, yarns, quality". Third edition, Bamberg: Meisenbach, 2004.

[3] Sadhan Chandraray. "Fundamentals and advances in knitting technology". Woodhead publishing limited. 2011.

[4] Jearranai L U and Tiluk D. " Quality Assurance in Circular Knitting, Part I: Theoretical Analysis", Thammasat International Journal of Science and Technology, Vol 4. 1999.

[5] A.S. Mrahat Khan. "Study On Weft Knitted Fabric Faults and Remedies", University Of Dhaka, College Of Textile Technology, Thesis, 2008.

[6] Saber Ben Abdessalem, etl. "Knitted Fabric Faults: Inspection, Causes and Solutions", Technology High School of Ksar Hellal,Textile research unit, Tunisia. 2009.

[7] Shady E., Gowayed Y., Abouiiana M., Youssef S. and Pastore C. "Detection and Classification of Defects in Knitted Fabric Structures", Textile Research Journal, 76(4),295-300, 2006.

[8] Zhang Y., Lu Z., Li J." Fabric Defect Detection and Classification Using Gabor Filters and Gaussian Mixture Model". In: Zha H., Taniguchi R., Maybank S. (eds) Computer Vision ACCV 2009. ACCV 2009. Lecture Notes in Computer Science, vol 5995. Springer, Berlin, Heidelberg. 2010.

[9] D. Brzakovic and H. Sari-Sarraf, "Automated Inspection of Nonwoven Web Materials: A Case Study", Proc. SPIE, vol. 2183. Feb. 1994.

[10] Kumar, A \& Pang, Grantham. "Defect Detection in Textured Materials Using Gabor Filters. Industry Applications, IEEE Transactions on. 38. 2002.

[11] Jing, J., Yang, P., Li, P., \& Kang, X. "Supervised Defect Detection on Textile Fabrics Via Optimal Gabor Filter". Journal of Industrial Textiles, 44(1), 4057. 2014.

[12] Han, Runping \& Zhang, Lingmin. "Fabric Defect Detection Method Based on Gabor Filter Mask". 2010 Second WRI Global Congress on Intelligent Systems. 3. 2009.

[13] Daugman, J.G. "Two-dimensional Spectral Analysis of Cortical Receptive Field Profiles". Vision Res. 20, 847856. 1980.

[14] Jaume Escofet, Rafael Fonolla Navarro, Maria Sagrario Millan Garcia- Verela, and Josep Mallofre Pladellorens., ’Detection of local Defects in Textile Webs Using Gabor Filters". Optical Engineering 37(8). 1998.

[15] C.Sujatha ; Selvathi, Dr. D. "An Optimal Solution For Image edge Detection Problem Using Simplified Gabor Wavelet". International Journal of Computer Science, Engineeringand Information Technology (IJCSEIT), 2 (2012), Nr. 3, S.

[16] Wei Jiang, Ting-Zhi Shen, Jian Zhang, Yu Hu, Xin-Yi Wang. " Gabor Wavelets for Image Processing". IEEE International Colloquium on Computing, Communication, Control, and Management, Vol.1. 2008.

[17] https://sebastianraschka.com/faq/docs/logisticregr-neuralnet.html.

[18] https: // en:wikipedia:org=wiki=Logisticregression.

[19] https://www.peterkovesi.com/matlabfns/PhaseCongruency/Docs/convexpl.html 